\title{
Evaluation of a Functional Model of Acquired Capability for Suicide in a Nationwide Sample of Korean Adults
}

\author{
Joon Deuk Lee', Maeng Je Cho², Jee Hoon Sohn², Subin Park², Sujeong Seong², \\ Joon Ho Ahn ${ }^{3}$, Bong-Jin Hahm², and Jin Pyo Hong ${ }^{\natural} \bowtie$ \\ ${ }^{1}$ Department of Psychology, Seoul National University, Seoul, Republic of Korea \\ ${ }^{2}$ Department of Psychiatry and Behavioral Science, Seoul National University College of Medicine, Seoul, Republic of Korea \\ ${ }^{3}$ Department of Psychiatry, University of Ulsan College of Medicine, Ulsan University Hospital, Ulsan, Republic of Korea \\ ${ }^{4}$ Department of Psychiatry, Samsung Medical Center, Sungkyunkwan University School of Medicine, Seoul, Republic of Korea
}

Objective We examined a functional model of acquired capability for suicide, which was elaborated from the "Interpersonal-Psychological Theory of Suicide".

Methods A total of 6,027 Korean community subjects were recruited from The Korean Epidemiologic Catchment Area study conducted in 2011. The subjects were assessed systematically using the Korean version of the Composite International Diagnostic Interview version 2.1, the Korean version of the Barratt Impulsiveness Scale, and the Korean version of the Mood Disorder Questionnaire. We used structural equation modeling to identify potential factors contributing to a suicide attempt.

Results Most aspects of the model were supported by the data and "the short-term enhancer for the acquired capability for suicide" had direct effects on suicide attempts. However, the suicidal planning effects of "the short-term enhancer for the acquired capability for suicide" were eliminated by the rule of parsimony.

Conclusion The main finding was that "the short-term enhancer for the acquired capability for suicide" is relevant to suicide attempts when it's direct, indirect, and reciprocal effects are tested within a more complete system of relationships than found in existing studies. The implications of these findings are discussed with regard to future conceptual work and empirical research.

Psychiatry Investig 2016;13(6):601-608

Key Words Suicide, Acquired capability, Suicidal ideation, Suicidal attempt, Model of suicide, Community sample.

\section{INTRODUCTION}

It is well known that not all people who have suicidal thoughts or ideation attempt suicide and that only a fraction of those who attempt suicide actually die. In response, Joiner ${ }^{1}$ claimed that "even if people desire to commit suicide, only those who are realistically capable in committing suicide end up dying due to suicide." As a possible answer, Joiner ${ }^{2}$ developed the "Interpersonal-Psychological Theory of Suicide-a functional model of the acquired capability for suicide" as an

Received: January 19, 2015 Revised: July 24, 2015

Accepted: August 3, 2015 Available online: November 2, 2016

$\triangle$ Correspondence: Jin Pyo Hong, MD, PhD

Department of Psychiatry, Samsung Medical Center, Sungkyunkwan University School of Medicine, 81 Irwon-ro, Gangnam-gu, Seoul 06351, Republic of Korea Tel: +82-2-3010-3421, Fax: +82-2-485-8381, E-mail: suhurhong@gmail.com (ac) This is an Open Access article distributed under the terms of the Creative Commons Attribution Non-Commercial License (http://creativecommons.org/licenses/bync/3.0) which permits unrestricted non-commercial use, distribution, and reproduction in any medium, provided the original work is properly cited. integrated model for suicide attempts. He said that suicide can be explained in three dimensions: 1) thwarted belongingness; 2) perceived ineffectiveness and resultant burdensomeness to others; and 3) the acquired capability for suicide.

Joiner et al. ${ }^{3}$ suggested that the acquired capability for suicide can act as a limiting factor to determine who among individuals that experience a serious desire for death by suicide will and will not die by suicide. However, suicide is a statistically very rare incident, and the act of suicide contradicts millions of years of evolutionary self-preservation programming. ${ }^{4}$ Therefore, this capability for suicide typically involves a long and difficult process that develops over time, and Joiner describes that acquired capability as a condition involving a heightened degree of fearlessness and pain insensitivity such that the action and ideas involved in suicide are no longer alarming. ${ }^{1}$ This kind of experience was described in Solomon's opponent process theory.

In addition to Joiner's theory, Smith and Cukrowicz ${ }^{5}$ argued 
that life experiences containing painful and provocative elements occurring in conjunction with the specific diatheses described earlier result in fearlessness of suicide and tolerance to the physical pain involved in suicide. The first point on vulnerability stems from research suggesting that suicide attempters, particularly violent attempters, experience reduced serotonergic activity. ${ }^{6,7}$ Low levels of serotonin have been associated with personality variables, such as aggression and impulsivity. ${ }^{7 m}$ pulsivity is a risk factor for suicide capability based, in part, on the fact that impulsive people are less likely to heed warning signs against self-harm. Of the vulnerabilities that stem from life events, the experience of physically painful and psychologically provocative life events is the most important. ${ }^{8}$ These experiences interact with the aforementioned dispositions, resulting in life threats or reduced warning signs against painful events, which, in turn, develop the capability of suicide. ${ }^{9} \mathrm{Hy}-$ pothetical experiences, such as mental rehearsal for suicide, can accelerate acquired skill or ability. ${ }^{1}$ Smith et al. ${ }^{9}$ claimed that these experiences in the form of acquired skills help reduce the fears of suicide and develop the strength to bear the physical pain when attempting suicide. In contrast, cultures or religions, such as Catholicism, that oppose suicide are a protective factor.

Joiner ${ }^{1}$ described the acquired capability for suicide as a static process that involves crossing of some idiosyncratic threshold after which the individual is perpetually capable of suicide. However, Smith et al. ${ }^{9}$ suggested that an individual can develop the capability for suicide in the moment via intoxication, psychosis, and so forth. This is the main difference between their suicide models. Smith et al. ${ }^{9}$ called the reinforcer "capability for suicide in the moment (short-term capability)". These actions or experiences that serve to reduce the perceptions of fear and sensations of pain involved in suicide in the short-term include substance intoxication, dissociative states, psychotic episodes, and manic episodes..$^{10-12}$

Therefore, the theoretical research of Smith et al. ${ }^{9}$ proposed a new concept in which the structural construction of the "the acquired capability for suicide" integrates a relatively time-stable factor as well as factors that can expedite the capability in the short-term. Furthermore, they have conceptualized not only serious and lethal suicide attempts, but also relatively less severe and successful suicide attempts. However, because the theoretical framework was proposed relatively recently, no study has validated the current model. The present experientially examined Smith et al. .9 "A functional model of the acquired capability component of the interpersonal-psychological theory of suicide".

Based on previous studies related to suicide and the acquired capability theory, the research topics of this study are: 1) Impulsivity, attitudes toward religion, pain and trauma experience as latent variables for acquired suicide capability. 2)
Manic symptoms, alcohol-related disorder, and psychosis as latent variables of short-term enhancers for acquired capability. Path hypotheses are as follows. 1) and 2) "Suicidal ideation" is positively related to "the short-term enhancer for the acquired capability and suicidal plan". 3) and 4) "Suicide plan" is positively related to "the short-term enhancer and the acquired capability for suicide". 5) "The short-term enhancer" is positively related to "the acquired capability for suicide". 6) "The acquired capability for suicide: is positively related to "suicide attempt".

\section{METHODS}

\section{Sample}

The Korean Epidemiologic Catchment Area (KECA) study was conducted in $2001^{13}$ and $2006^{14}$ using the Korean version of the Composite International Diagnostic Interview 2.1 (KCIDI 2.1) ${ }^{15} \mathrm{~A}$ follow-up to the KECA study was conducted in 2011. This follow-up was designed to determine the lifetime and 12-month prevalence, socioeconomic correlates, and comorbidities of major mental disorders in the Diagnostic and Statistical Manual of Mental Disorders, Fourth Edition (DSMIV) among Korean adults. Subjects were selected using a stratified, multi-stage, clustered sample design, based on a population census conducted by community registry offices in 2010 . One individual per selected household, with the earliest birthday, with reference to the day of the birth month, was chosen randomly. From an initially selected 8,196 subjects (age, 18-74 years), 6,027 face-to-face interviews ( $73.5 \%$ response rate) were conducted.

Seventy-eight interviewers were recruited from each catchment area for the 78 interview. These interviewers were psychiatric nurses, social workers, and medical students, all of whom were familiar with psychiatric epidemiologic surveys. The three psychiatrists who served as trainers were certified by the Composite International Diagnostic Interview training center at the University of Michigan (USA). All interviewers participated in a 5-day training session that included didactic sessions to review general interview skills and the interview instrument, mock interviews, and role-playing exercises. ${ }^{16,17}$ Trainers monitored interviews on closed-circuit television and provided feedback.

\section{Assessment}

\section{Korean version of the Composite International Diagnostic Interview}

Trained lay interviewers administered the K-CIDI ${ }^{15}$ to each subject. The $\mathrm{CIDI}^{18}$ is a fully structured diagnostic modality designed to establish psychiatric diagnoses based on the defi- 
nitions and criteria in the DSM-IV. ${ }^{19}$ The K-CIDI was developed according to World Health Organization (WHO) guidelines. ${ }^{20}$ The inter-rater reliability, test/retest reliability, and validity of the K-CIDI showed kappa values of 0.86-1.00, $0.42-0.89$, and $0.50-1.00$, respectively. In the present study, we analyzed the relationships between exposure to childhood trauma and lifetime prevalence of specific psychiatric disorders. Each variable containing a missing value was estimated using the maximum likelihood method (MLM).

\section{Religious attitudes}

A demographics item (DE3) in the CIDI interview was used to explore religious attitudes. We hypothesized that the responders' positive attitude about their religion was negatively correlated with suicide attempts. Religious attitudes were assessed with a single question: "How important is religion to you (if you have a religion)?" Religious attitude were evaluated using quartiles of one (not at all important) to four (very important).

\section{Pain experience}

Using the Pain disorder section of the K-CIDI, chronic pain experience (at any time during life) was assessed, including abdominal pain, back pain, arthralgia, limb pain, chest pain, headache, menstrual pain, dysuria, penile pain, and any other pain. Each pain experience was counted as 1 point, and the sum of the total score was recorded (0-10).

\section{Traumatic event experience}

Using the Post-Traumatic Stress Disorder section of the KCIDI, exposure to the following post-traumatic events (at any time during life) was initially assessed, including military combat, sudden injury/accident, natural disaster, seeing someone hurt or killed, rape, sexual assault, physical assault, threat/kidnapping, torture, or other traumatic event. The subject's age at the time of the event was also recorded. Each traumatic experience was counted as 1 point, and the sum of the total score was recorded (0-12).

\section{Suicidality}

We used the K-CIDI module on suicide to assess lifetime suicidality, including suicidal ideation, suicidal plans, and suicide attempts. These questions were used in the WHO-Suicide Prevention Multisite Intervention Study on Suicidal Behaviors. ${ }^{21-23}$ Suicidal ideation was assessed with the following question: "Have you ever seriously thought about committing suicide?" Existence of a suicide plan was assessed with the following question: "Have you ever concretely planned suicide?" Suicide attempts were assessed with the following question: "Have you ever attempted suicide?" Information on age at the time of the first suicide attempt and number of suicide attempts was also collected.

\section{Korean version of the Barratt Impulsiveness Scale}

The BIS was translated to Korean by Lee, ${ }^{24}$ and we used the standardized BIS 11. The BIS is comprised of 23 items divided into three subcategories: nine questions pertain to unplanned/ impulsivity, eight to motor impulsivity, and six to cognitive impulsivity. The internal consistencies determined in a previous study that used the impulsivity scale in terms of unplanned/ impulsivity, motor impulsivity, and cognitive impulsivity were $0.30,81$, and 0.51 , respectively. The current study's total internal consistency was 0.86 .

\section{Korean version of the Mood Disorder Questionnaire}

Hirschfeld et al. ${ }^{25}$ developed the MDQ to distinguish bipolar disorder. This self-report questionnaire was developed based on the DSM-IV criteria for bipolar disorder in which in criterion 1 of the 13 items they must report "yes" to at least seven. These items must appear at the same time for criterion 2, and significant impairment must be experienced due to these symptoms for criterion 3 . Therefore, if all three criteria are satisfied, bipolar spectrum disorder is concluded. However, the K-MDQ was translated and standardized by Jon et al. ${ }^{26}$ and unlike the original questionnaire, criteria 2 and 3 have been omitted, and bipolar disorder is determined by a minimum score of 7 on criterion 1 .

\section{Latent variables}

\section{Acquired capability for suicide}

The variables that comprise the acquired capability for suicide are: attitudes toward religion (sociocultural background of suicide), pain experience (habituation of distress), traumatic experience (habituation of psychological distress), and impulsivity (dispositional factors).

\section{Short-term enhancer (of acquired capability for suicide)}

Latent variables that may temporarily increase the acquired capability for suicide have been assumed, and alcohol use disorder and manic symptoms (K-MDQ) of the variables were measured.

\section{Statistical analysis}

Descriptive statistics are used for the entire sample with SPSS 18.0 software (SPSS Inc., Chicago, IL, USA). A structural equation model (SEM) analysis was performed with AMOS 18.0. The SEM analysis is used to assess the causal relationships among exogenous, mediator, and criterion variables simultaneously. ${ }^{27,28}$ We organized the SEMs, which consisted of 
observed and latent variables, using AMOS 18.0 software, and then calculated the maximum likelihood estimate for the model parameters using covariance of the total sample. Goodness of fit of the data for the proposed model was assessed with chi-square indices $\left(\chi^{2}\right)$, the normed fit index (NFI), the comparative fit index (CFI), and the root mean square error of approximation (RMSEA).

\section{RESULTS}

\section{Sociodemographic characteristics of the subjects}

A total of 6,022 respondents were included (2,308 males and 3,714 females; male: female ratio, $1: 1.61$. In total, 4,894 respondents (1,910 males and 2,984 females) were $<64$ years. A 12year education was the most common with 2,161 respondents and 1,694 respondents reported being unemployed. A total of 3,494 respondents reported having a religion and the majority of the respondents lived in an urban district (Table 1).

The clinical characteristics of the respondents were suggested by constructing three latent variables from the observed variables. As results, mean BIS scale score was 45.19 [standard deviation $(\mathrm{SD})=0.18]$, and the means of each religious attitude are shown in Table 2. A total of 1,143 (19\%) respondents reported chronic pain symptoms and 1,556 (25.8\%) reported traumatic experiences. Mean MDQ score was $20.96(\mathrm{SD}=0.01)$. A total of 647 (10.7\%) respondents had an alcohol-related dis-

Table 1. Socio-demographic characteristics of subjects

\begin{tabular}{lc}
\hline \multicolumn{1}{c}{ Variables } & $\mathrm{N}(\%)$ \\
\hline Sex & \\
Male & $2,308(38.3)$ \\
Female & $3,714(61.7)$ \\
Age & \\
$\quad$ Mean (SD) & $47.88( \pm 15.3)$ \\
Education (years) & \\
0 & $235(3.9)$ \\
$1-6$ & $902(15.0)$ \\
$7-9$ & $714(11.9)$ \\
$10-12$ & $2,010(33.4)$ \\
13 or more & $2,161(35.9)$ \\
Employment status & \\
Unemployed & $1,694(28.2)$ \\
Religion & \\
Yes & $3,493(58.4)$ \\
No & $2,490(41.3)$ \\
Area of residence & \\
Urban & \\
Rural & $4,687(77.8)$ \\
\hline
\end{tabular}

order, 34 (0.6\%) suffered from psychosis, 951 (15.8\%) had experienced suicidal ideation, $210(3.5 \%)$ had a suicide plan, and 201 (3.3\%) had attempted suicide (Table 2).

\section{Evaluation of proposed models}

SEM was used to assess the relationships between the suicidal ideation, suicide attempts, and other related factors of the acquired capability for suicide for the Korean community sample based on the factor analysis results of the combined data. SEM is a technique to simultaneously estimate the relationships between observed and latent variables (measurement model) and the relationships between latent variables (structural model). SEM has gained popularity because it combines confirmatory factor analysis and regression analysis to model a variety of psychological, sociological, and other relationships.

We designed the SEM to examine the relationships between latent and observed variables. Our primary purpose was to identify the existence of mediating variables that were not

Table 2. Clinical characteristics of subjects

\begin{tabular}{clr}
\hline \multicolumn{1}{c}{ Variables } & Measurement & \multicolumn{1}{c}{ Values } \\
\hline $\begin{array}{c}\text { Acquired capability for suicide } \\
\text { Barratt impulsiveness scale } \\
\text { (BIS) }\end{array}$ & Mean (SD) & $45.19( \pm 0.18)$ \\
Religious attitude & Mean (SD) & $2.35( \pm 0.16)$ \\
& Very important & $1,646(27.3 \%)$ \\
& Important & $1,518(25.2 \%)$ \\
& Not important & $1,529(26.7 \%)$ \\
& Not at all & $1,038(18.1 \%)$ \\
& important & \\
Sum of pain & Mean (SD) & $0.41( \pm 0.01)$ \\
& None & $4,875(81 \%)$ \\
& 1 symptom & $1,143(19 \%)$ \\
& or more & \\
Sum of trauma & Mean (SD) & $0.41( \pm 0.01)$ \\
& None & $4,461(74.1 \%)$ \\
& 1 trauma & $1,556(25.8 \%)$ \\
& or more &
\end{tabular}

Short-term enhancer

Mood disorder questionnaire Mean (SD)

$20.96( \pm 0.27)$ (MDQ)

\begin{tabular}{lcc}
$\begin{array}{l}\text { Alcohol related disorder } \\
\text { Psychosis* }\end{array}$ & Yes & $647(10.7 \%)$ \\
$\begin{array}{l}\text { Suicidal behaviors } \\
\text { Suicidal idea }\end{array}$ & Yes & $34(0.6 \%)$ \\
Suicidal plan & Yes & $951(15.8 \%)$ \\
Suicidal attempt & Yes & $210(3.5 \%)$ \\
\hline
\end{tabular}

*schizophrenia spectrum disorder including in schizophrenia, schizoaffective disorder, and delusional disorder 
studied clearly in the "acquired capability for suicide model". Based on a literature review, we designed a causal relationship model between suicidal ideation and suicide attempts. We then added and tested the relationships between "the acquired capability for suicide", "the short-term enhancer of acquired capability", and "suicide plan" as multiple mediating variables.

The proposed model hypothesized that significant causal relationships exist between two latent constructs of "the acquired capability for suicide", "the short-term enhancer for acquired capability", and three observed variables, such as "suicide ideation", "suicide plan", and "suicide attempt". The causal relationships represented the six hypotheses in the path model (Figure 1). Empirical evaluation of such hypotheses is complicated by the fact that latent constructs are not directly observable. The evaluation was based on sets of observed or measured variables that serve as indicators of latent variables, and the relationships between the observed and latent variables were estimated using factor analysis.

As a result, all estimated factor loadings were significantly related between the latent and observed variables (Table 3). We detected positive relationships for all variables, except religious attitudes. This result supports our premise that religious attitudes are a protective factor for suicide attempts.

All path coefficients were significantly related with each other. Path hypotheses 1 and 2 (i.e., "suicidal idea" is positively related to " the short-term enhancer for the acquired capability and suicidal plan") were supported at an optimal level with a standardized estimate of $0.431(\mathrm{p}<0.001)$ and a standardized estimate of 0.504 ( $\mathrm{p}<0.001)$. Path hypotheses 3 and 4 (i.e., "suicide plan" is positively related to "the short-term enhancer and the acquired capability for suicide") was supported with a standardized estimate of $0.100(\mathrm{p}<0.013)$ and a standardized estimate of $0.684(\mathrm{p}<0.001)$. Path hypothesis 5 (i.e., "the shortterm enhancer" is positively related to "the acquired capability for suicide") was supported with a standardized estimate of 0.596 ( $<<0.001)$. Path hypothesis 6 (i.e., "the acquired capability for suicide" is positively related to "suicide attempt") was supported at a standardized estimate of 0.690 (p<0.001) (Table 4).

We adopted the SEM model comparison method as a model selection strategy. First, we added a modified model to the original model and calculated the difference in model fit. Our modified model was a nested model in which a pathway was

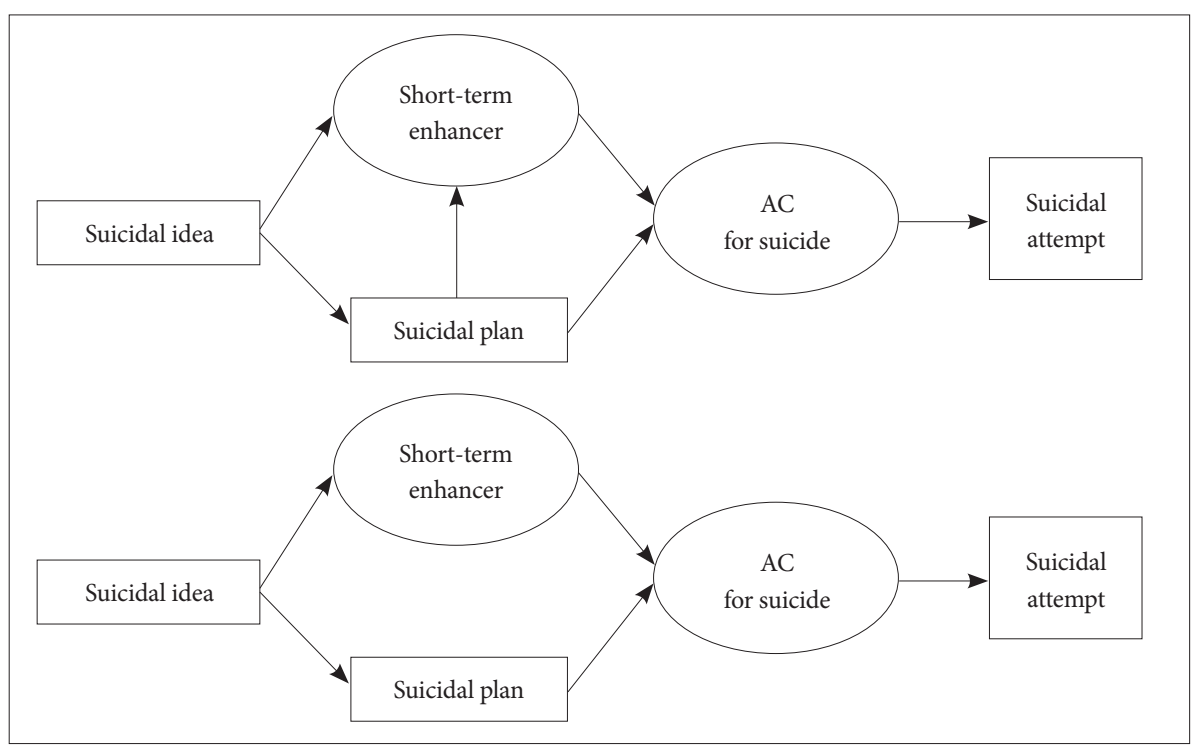

Figure 1. Comparison between the original model (including the pathway from suicide plan to short-term enhancer) and the modified model (not including that pathway). AC: Acquired Capability.

Table 3. Estimation of relationship between observed variables and latent variables

\begin{tabular}{|c|c|c|c|c|c|c|}
\hline Latent variable & Observed variable & Estimate & Standardized estimate & S.E. & C.R. & p-value \\
\hline Acquired capability & Sum pain & 1 & 0.192 & & & $* * *$ \\
\hline \multirow[t]{3}{*}{ for suicide } & Sum trauma & 1.165 & 0.259 & 0.106 & 10.97 & $* * *$ \\
\hline & BIS & 3.647 & 0.075 & 1.02 & 3.57 & $* * *$ \\
\hline & Religious attitude & -0.306 & -0.060 & 0.079 & -3.89 & $* * *$ \\
\hline \multirow[t]{3}{*}{ Short-term enhancer } & Alcohol & 1 & 0.272 & & & $* * *$ \\
\hline & psychosis & 0.126 & 0.142 & 0.019 & 6.55 & $* * *$ \\
\hline & MDQ & 49.225 & 0.308 & 5.930 & 8.30 & $* * *$ \\
\hline
\end{tabular}

${ }_{* * *} \mathrm{p}<0.001$. BIS: barratt impulsiveness scale, MDQ: mood disorder questionnaire 
removed from the original model (Figure 1). Model fit determines the degree to which the SEM fits the sample data. ${ }^{29}$ As a result, all confirmatory factor analyses (CFAs) of constructs and path analyses produced a relatively good fit, as indicated by the goodness of fit indices, such as CFI $>0.90$ and a RMSEA value $<0.08 .^{30}$ The results of the absolute measures for the original and modified models clearly surpassed the cut-off values, confirming that the structural model highly fit the sample data (original model: $\chi^{2}=389.996, \mathrm{df}=33, \mathrm{NFI}=0.924$, CFI $=0.930$, RMSEA=0.042; modified model: $\chi^{2}=393.204, \mathrm{df}=34, \mathrm{NFI}=$ 0.923, CFI=0.929, RMSEA=0.042). We used the MLM to estimate the parameters.

We examined the chi-square difference test to compare the original and modified models (Figure 1). Because the modified model is nested in the original model, a chi-square difference test can be applied to determine relative parsimony. According to the model fit analysis, the difference between the two models was $3.208(\Delta \mathrm{df}=1)$, which is smaller than 3.84 $\left(0.95 \chi^{2} 2\right)$. Therefore, the modified model was selected by the rule of parsimony as the final model, and the path from suicide plan to short-term enhancer was eliminated. This result seems reasonable because the short-term enhancer has an indiscreet and reckless property. Thus, we accepted our modified model as the final model (Figure 2).

Previous studies have supported a causal relationship between "the acquired capability for suicide" and "suicide attempts" in patients with a psychiatric disorder. ${ }^{31-33}$ Joiner's $^{1}$ concept of the "acquired capability for suicide" has gradual and intentional growth by (internal) psychological rehearsal or (external) physical exposure. However, Smith et al. ${ }^{9}$ added the variable, "short-term bolstering of the capability for suicide during a suicide attempt'(we call it "short-term enhancer" in this study). This variable could be an explanation for non- intentional suicide attempt. In this context, our finding of no causal relationship between suicide planning and short-term enhancer may be a more plausible result than that of the original model.

\section{DISCUSSION}

We examined "a functional model of the acquired capability for suicide" and our data support their conceptual model. In our model, the short-term enhancer, as a latent variable, was also supported empirically, which is our most important finding. In our study, short-term enhancers may have been a useful concept to evaluate unintentional and unplanned suicide attempts. Further research in this field is needed to discuss integrating short-term enhancers for the acquired capability theory. It is a very important finding that Smith and Cukrowicz's model was supported with an optimal level of model fit in a large Korean communuity sample. Therefore, preventing suicide should have different interventions between those who have specific suicide plans and others who have drug addictions, psychotic episodes, or manic episodes. ${ }^{10-12}$

Our study holds additional significance beyond the aforementioned findings. First, as a nation that has received attention due to its high suicide rate, we explored the psychological factors that may influence suicidal ideation leading to an actual suicide attempt in a large community sample through the SEM. This study was made possible with exemplary data because of expansion of the representative sample due to acquisition of national data. The 2011 Korean Mental Illness Epidemiologi-

Table 4. Estimation of path coefficients

\begin{tabular}{lccccc}
\hline \multicolumn{1}{c}{ Path } & Estimate & Standardized estimate & S.E. & C.R. & p-value \\
\hline Suicidal idea $\rightarrow$ suicidal plan & 0.217 & 0.431 & 0.006 & 36.919 & $* * *$ \\
Suicidal idea $\rightarrow$ STE & 0.116 & 0.504 & 0.009 & 12.292 & $* * *$ \\
STE $\rightarrow$ AC for suicide & 1.485 & 0.596 & 0.165 & 9.001 & $* * *$ \\
Suicidal plan $\rightarrow$ STE & 0.045 & 0.100 & 0.018 & 2.479 & 0.013 \\
Suicidal plan $\rightarrow$ AC for suicide & 0.779 & 0.684 & 0.059 & 13.113 & $* * *$ \\
AC for suicide $\rightarrow$ suicidal attempt & 0.592 & 0.690 & 0.045 & 13.245 & $* * *$ \\
\hline
\end{tabular}

*** $p<0.001$. STE: Short-term Enhancer, AC: Acquired Capability

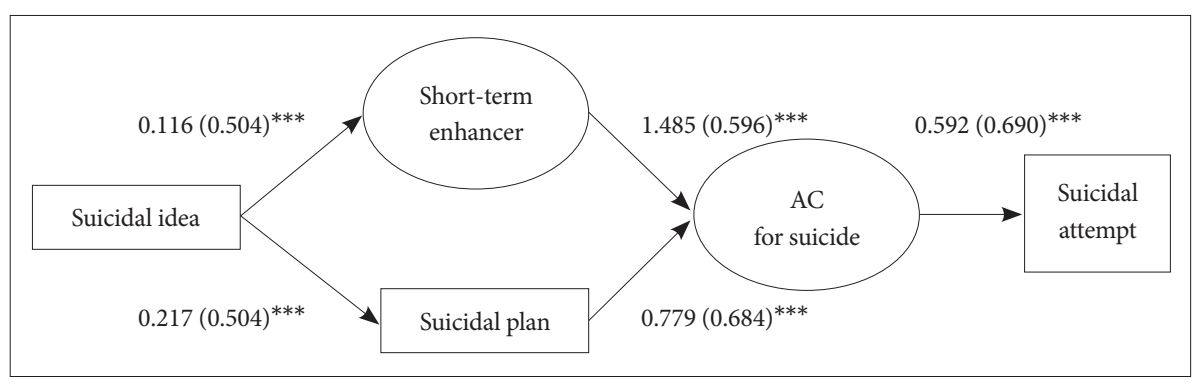

Figure 2. Final structural model of the constructs for suicidal behavior, including standardized path coefficients and levels of significance $\left({ }^{* * *} p<0.001\right)$. AC: Acquired Capability. 
cal Investigation allowed inclusive and strict data to be acquired to measure sociocultural backgrounds in relation to suicide, mental illnesses that increase the possibility of suicide attempts, subjective experiences of trauma and pain, impulsivity, and manic tendencies. Beyond lethal suicide attempts, models related to unsuccessful suicide attempts were also explored. Kessler et al. ${ }^{34}$ referred to the 2001-2003 American National Comorbidity Survey, in which 3.3\% of Americans reported suicidal ideation but the actual percentage of who attempted suicide was only $42.5 \%$. In contrast, those who had attempted suicide previously reported more suicide-related symptoms, ${ }^{35}$ with greater chances of more lethal suicide attempts. ${ }^{36}$ Joiner $^{2}$ responded to this phenomenon by developing the "Interpersonal-Psychological Theory of Suicide-a functional model of the acquired capability for suicide" as an integrated model for suicide attempts. Joiner ${ }^{1}$ claimed that painful or stimulating experiences, such as physical violence, self-injury, and substance abuse, can indirectly help develop suicidal capability, even if such experiences are not explicitly related to an actual suicidal attempt. In agreement with Joiner's claim, many studies have shown that substance abuse, prostitution, and physically violent activities are related to suicide attempts and death due to suicide and are therefore contributing factors that heighten the danger of suicide. ${ }^{37-39}$ While Joiner's1 the model aims to predict serious and lethal suicide attempts, Smith et al'.s ${ }^{9}$ model aims to conceptualize nonlethal suiciderelated behaviors. Therefore, an empirical study that examines experiential data of unsuccessful suicide attempters within an inclusive model increases the overall understanding of suicidal behavior. Although such attempters did not succeed in committing suicide, distinguishing these people who may show progressive danger early on, is useful.

Although this study supports our proposed model with a good model fit, the limitations include weak homogeneity on the religious attitude sub-scale. Previous studies mentioned that religion affects suicide, particularly the "Catholic tradition" which has the strictest dogma regarding suicide. ${ }^{5}$ However, our "religious attitude" item included Catholicism, Buddhism, Protestantism, Protestant Christianity, and other religions. The second limitation was only including the sample of community-dwelling individuals, as particular populations have a higher prevalence of mental health problems. Third, most of the diagnostic information was based on life-time prevalence; therefore, differences based on age were not considered. Fourth, sex differences in the acquired capability for suicide were not explored. Previous studies have reported that males acquire the capability for suicide more frequently than that of females, and males report greater sensation-seeking tendencies. ${ }^{40}$ Fifth, the relationship between capability for suicide and an actual measured scale was not executed (e.g., Na- demin et al.s ${ }^{42}$ the "acquired capability for suicide scale"). Sixth, we could not include all variables related to suicide (e.g., neurological process, abuse history, accidental experience, profession, etc). Due to the physical limitations of such a large-scale interview, we omitted some important variables because our data set is a part of product from the nationwide mental health program. At the time (2009-2010) that mental health program had designed, Smith et al'.s ${ }^{9}$ model was not published. However, we believed that the crucial observed variables can support the conceptual model in our study. Finally, our data were crosssectional. In fact, the cross-sectional nature of the study was a limitation, as we could not establish definite causal relationships. We attempted to examine the possibility of suicide attempts via the impulsive pathway.

In summary, we verified Smith et al.s 9 "functional model of the acquired capability for suicide." Notwithstanding the limitations, this study holds significance as the first large-scale community sample in which statistics supported the model. Because the data were acquired previously, new variables were unable to be introduced. However, the goodness-of-fit of the model was sufficient for future investigations. It is hoped that future studies do not merely gather data in terms of mental health and suicide but rather investigate the direction of a more developed and structured model.

\section{Acknowledgments}

This study was funded by the Korean Ministry of Health and Welfare. The authors wish to express gratitude to 12 local investigators and 79 interviewers. This study was supported by the Korea Healthcare Technology R\&D project, Ministry of Health \& Welfare, Republic of Korea (grant number A120051).

\section{REFERENCES}

1. Joiner TE Jr. Why People Die by Suicide? Cambridge, MA: Harvard University Press; 2005.

2. Joiner TE, Van Orden KA. The Interpersonal-psychological theory of suicidal behavior indicates specific and crucial psychotherapeutic targets. Int J Cogn Ther 2008;1:80-89.

3. Joiner TE Jr, Conwell Y, Fitzpatrick KK, Witte TK, Schmidt NB, Ber$\lim$ MT, et al. Four studies on how past and current suicidality relate even when "everything but the kitchen sink" is covaried. J Abnorm Psychol 2005;114:291-303.

4. Ohman A, Mineka S. Fears, phobias, and preparedness: toward an evolved module of fear and fear learning. Psychol Rev 2001;108:483522.

5. Smith PN, Cukrowicz KC. Capable of suicide: a functional model of the acquired capability component of the Interpersonal-Psychological Theory of Suicide. Suicide Life Threat Behav 2010;40:266-275.

6. Arango V, Underwood MD, Gubbi AV, Mann JJ. Localized alterations in pre- and postsynaptic serotonin binding sites in the ventrolateral prefrontal cortex of suicide victims. Brain Res 1995;688:121-133.

7. Joiner TE Jr, Brown JS, Wingate LR. The psychology and neurobiology of suicidal behavior. Annu Rev Psychol 2005;56:287-314.

8. Van Orden KA, Witte TK, Gordon KH, Bender TW, Joiner TE Jr. Suicidal desire and the capability for suicide: tests of the interpersonalpsychological theory of suicidal behavior among adults. J Consult Clin Psychol 2008;76:72-83. 
9. Smith PN, Cukrowicz KC, Poindexter EK, Hobson V, Cohen LM. The acquired capability for suicide: a comparison of suicide attempters, suicide ideators, and non-suicidal controls. Depress Anxiety 2010;27:871877.

10. Brown GK, Beck AT, Steer RA, Grisham JR. Risk factors for suicide in psychiatric outpatients: a 20-year prospective study. J Consult Clin Psychol 2000;68:371-377.

11. Kessler RC, Borges G, Walters EE. Prevalence of and risk factors for lifetime suicide attempts in the National Comorbidity Survey. Arch Gen Psychiatry 1999;56:617-626.

12. Zoroglu SS, Tuzun U, Sar V, Tutkun H, Savacs HA, Ozturk M, et al. Suicide attempt and self-mutilation among Turkish high school students in relation with abuse, neglect and dissociation. Psychiatry Clin Neurosci 2003;57:119-126.

13. Cho MJ, Kim JK, Jeon HJ, Suh T, Chung IW, Hong JP, et al. Lifetime and 12-month prevalence of DSM-IV psychiatric disorders among Korean adults. J Nerv Ment Dis 2007;195:203-210.

14. Cho MJ, Chang SM, Lee YM, Bae A, Ahn JH, Son J, et al. Prevalence of DSM-IV major mental disorders among Korean adults: a $2006 \mathrm{Na}$ tional Epidemiologic Survey (KECA-R). Asian J Psychiatr 2010;3:26-30.

15. Cho MJ, Hahm BJ, Suh DW, Hong JP, Bae JN, Kim JK, et al. Development of a Korean Version of the Composite International Diagnostic Interview (K-CIDI). J Korean Neuropsychiatr Assoc 2002;41:123-137.

16. World Health Organization. CIDI, Core Version 2.1 Interviewr's Manual. Geneva: World Health Organization; 1997.

17. World Health Organization. CIDI, Core Version 2.1 Trainer's Manual. Geneva: World Health Organization; 1997.

18. World Health Organization. Composite International Diagnostic Interview (CIDI), Version 1.0. Geneva: World Health Organization; 1990.

19. Amerian Psychiatric Association. Diagnostic and Statistical Mannual of Mental Disorders. 4th Edition. Washington, DC: Amerian Psychiatric Association; 2000

20. World Health Organization. Procedures for the Development of New Language Versions of the WHO Composite International Diagnostic Interview (WHO-CIDI). Geneva: World Health Organization; 1997.

21. Bertolote JM, Fleischmann A. Suicidal behavior prevention: WHO perspectives on research. Am J Med Genet C Semin Med Genet 2005;133C: $8-12$.

22. Botega NJ, Barros MB, Oliveira HB, Dalgalarrondo P, Marin-Leon L. Suicidal behavior in the community: prevalence and factors associated with suicidal ideation. Rev Bras Psiquiatr 2005;27:45-53.

23. Fleischmann A, Bertolote JM, De Leo D, Botega N, Phillips M, Sisask $M$, et al. Characteristics of attempted suicides seen in emergency-care settings of general hospitals in eight low- and middle-income countries. Psychol Med 2005;35:1467-1474.

24. Lee HS. Patterns of impulsiveness. J Humanit 1994;21:93-104.

25. Hirschfeld RM, Holzer C, Calabrese JR, Weissman M, Reed M, Davies $\mathrm{M}$, et al. Validity of the mood disorder questionnaire: a general popu- lation study. Am J Psychiatry 2003;160:178-180.

26. Jon DI, Hong N, Yoon BH, Jung HY, Ha K, Shin YC, et al. Validity and reliability of the Korean version of the Mood Disorder Questionnaire. Compr Psychiatry 2009;50:286-291.

27. Hatcher L. A Step-by-Step Approach to Using the SAS System for Factor Analysis and Structural Equation Modeling. Cary, NC: SAS Institute; 2003.

28. Kline RB. Principles and Practice of Structural Equation Modeling. New York: Guilford Press; 1998.

29. Schumacker RE, Lomax RG. A Beginner's Guide to Structural Equation Modeling. Mahwah, NJ: L. Erlbaum Associates; 1996.

30. Hair JF. Multivariate Data Analysis (7th Ed). Upper Saddle River, NJ: Prentice Hall; 2010.

31. Bryan CJ, Cukrowicz KC. Associations between types of combat violence and the acquired capability for suicide. Suicide Life Threat Behav 2011;41:126-136.

32. Bryan CJ, Cukrowicz KC, West CL, Morrow CE. Combat experience and the acquired capability for suicide. J Clin Psychol 2010;66:1044-1056.

33. Witte TK, Didie ER, Menard W, Phillips KA. The relationship between body dysmorphic disorder behaviors and the acquired capability for suicide. Suicide Life Threat Behav 2012;42:318-331.

34. Kessler RC, Berglund P, Borges G, Nock M, Wang PS. Trends in suicide ideation, plans, gestures, and attempts in the United States, 1990-1992 to 2001-2003. JAMA 2005;293:2487-2495.

35. Rudd MD, Joiner T, Rajab MH. Relationships among suicide ideators, attempters, and multiple attempters in a young-adult sample. J Abnorm Psychol 1996;105:541-550.

36. Joiner TE Jr, Steer RA, Brown G, Beck AT, Pettit JW, Rudd MD. Worstpoint suicidal plans: a dimension of suicidality predictive of past suicide attempts and eventual death by suicide. Behav Res Ther 2003;41:14691480 .

37. Darke S, Ross J. Suicide among heroin users: rates, risk factors and methods. Addiction 2002;97:1383-1394.

38. Kidd SA, Kral MJ. Suicide and prostitution among street youth: a qualitative analysis. Adolescence 2002;37:411-430.

39. Whitlock FA, Broadhurst AD. Attempted suicide and the experience of violence. J Biosoc Sci 1969;1:353-368.

40. Anestis MD, Bender TW, Selby EA, Ribeiro JD, Joiner TE. Sex and emotion in the acquired capability for suicide. Arch Suicide Res 2011;15:172182.

41. Witte TK, Gordon KH, Smith PN, Van Orden KA. Stoicism and sensation seeking: male vulnerabilities for the acquired capability for suicide. J Res Pers 2012;46:384-392.

42. Nademin E, Jobes DA, Pflanz SE, Jacoby AM, Ghahramanlou-Holloway $\mathrm{M}$, Campise R, et al. An investigation of interpersonal-psychological variables in air force suicides: a controlled-comparison study. Arch Suicide Res 2008;12:309-326. 